\title{
Wearable PPG Sensor with Bluetooth Data Transmission for Continual Measurement in Low Magnetic Field Environment
}

\author{
Jiří Přibil \\ Department of Imaging Methods \\ Institute of Measurement Science \\ Slovak Academy of Sciences \\ Bratislava, Slovak Republic \\ Jiri.Pribil@savba.sk
}

\author{
Anna Přibilová \\ Department of Biomeasurements \\ Institute of Measurement Science \\ Slovak Academy of Sciences \\ Bratislava, Slovak Republic \\ Anna Pribilova@savba.sk
}

\author{
Ivan Frollo \\ Department of Imaging Methods \\ Institute of Measurement Science \\ Slovak Academy of Sciences \\ Bratislava, Slovak Republic \\ Ivan.Frollo@savba.sk
}

\begin{abstract}
The paper devotes to a developed wearable photoplethysmographic (PPG) sensor for human heart pulse monitoring. The sensor can work in a weak magnetic field with radiofrequency (RF) disturbance present during patient examination in the low field magnetic resonance imaging device. The experiments have confirmed the proper real-time functioning with safe wireless communication and data transmission between the sensor and the control device performing post-processing of the PPG signal and estimation of the signal properties (level threshold, ripple, range, etc.). The developed control application for Windows platform performs also signal filtering and heart rate (HR) determination. Influence of the used sampling frequency on the precision and stability of the finally determined HR values was analyzed, too.
\end{abstract}

Keywords - photoplethysmographic wave, wearable sensor, signal measurement and processing, magnetic resonance tomograph

\section{INTRODUCTION}

Rapid alteration of the gradient-coil current causes the vibration and the acoustic noise radiating from the magnetic resonance imaging (MRI) system with possible impact on patients as a reversible hearing threshold shift [1]. One of the MRI applications includes observation of dynamic changes in the vocal tract geometry during human voice phonation [2]. In such a situation, the stress may evoke the tension of the vocal cords and the change of the $3 \mathrm{D}$ shape of the vocal tract of an examined person leading to the modification of the speech signal. The main aim of our present work is the monitoring of this stress by the blood pressure (BP) and the heart rate (HR) evaluation.

The mental stress can effectively be identified by the photoplethysmography (PPG) signal [3]. We have already used the PPG measurement of the HR to monitor the physiological impact of the noise on a person examined inside the MRI scanning device [4]. In this measurement, the PPG sensors, the control units, and the storage devices were interconnected by cables leading from the shielding cage of the MRI device. It was uncomfortable to he tested person and the realization of real-time measurement was rather difficult.

The motivation of this research was necessity of a wearable device enabling wireless PPG sensing with Bluetooth (BT) data transfer [5] to the recording device. In the frame of the performed practical experiments, the basic functions of the developed PPG sensor as well as the built control application were stepwise tested. The auxiliary measurement consists of real-time sensing, transmission, and

This work was funded by the projects VEGA2/0003/20, COST

CA16116, APVV-19-0032, and APVV-19-0531. storage operations (including signal filtering and HR value determination) - in the normal laboratory conditions. The main experiment with the PPG signal sensing inside the running MRI device has shown that the PPG signal must be filtered and all PPG sensor parts must be shielded to avoid strong radiofrequency (RF) disturbance in the scanning area.

\section{SUBJECT AND METHODS}

\section{A. PPG Sensors - Basic Principles and Types}

There exist two modes of optical sensors for PPG signal measurement: transmission and reflection. Both sensor types consist of two basic parts: a transmitter (light source - LS) and a receiver (photo detector - PDT). The arrangement of one or more light emitting diodes (LEDs) and one or more PDTs elements depends on the sensor operation mode. In the transmission mode, the LEDs and PDTs are placed on the opposite sides of the measured human tissue. In the reflection PPG sensor, the PDTs and LEDs are placed on the same skin surface. The recorded PPG signal contains two distinguished pulse wave parts (systolic and diastolic) that can be picked up from ears, fingers, or wrists of both upper limbs mainly using the optical sensors working on the transmission principle [6].

Three basic parts compose a wearable PPG sensor for real-time signal pick-up: (1) basic analog circuits with LS and PDT elements integrated on a board, (2) a processor for signal management including an analog to digital (A/D) converter and a universal serial communication interface (USART), (3) a Wi-Fi or BT receiver/transmitter for communication with external devices - see the diagram in Fig. 1. Galvanic connection with other devices powered by an $\mathrm{AC}$ line is prevented by using batteries for the power supply of the sensor. The sensor can work in slave or master mode depending on the sensing and transmission strategy.

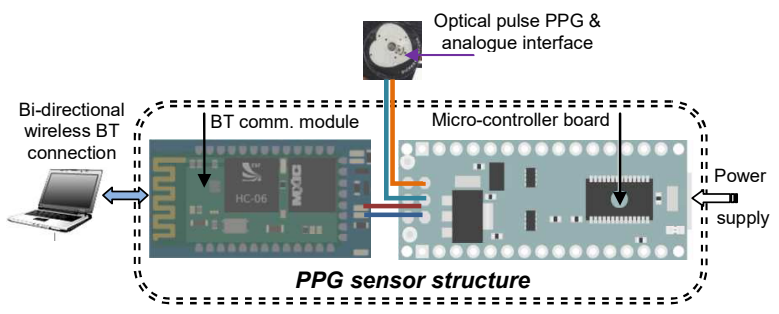

Fig. 1. Principal structure diagram of a wearable PPG sensor.

\section{B. Determination of PPG Signal Properties}

The PPG cycle frequency corresponding to the heart rate varies in healthy humans within the range from 60 to 
$100 \mathrm{~min}^{-1}$. It means that the frequency $f_{\mathrm{s}}$ of about $150 \mathrm{~Hz}$ seems to be sufficient for the PPG signal sampling. The amplitude of the picked-up PPG signal is usually not constant (with typical signal modulation) and it can be often partially disturbed or degraded. Therefore, the sensed raw PPG signal must be smoothed before the HR determination. A smoothing method suitable for real-time processing of this relatively slow signal is a moving average (MA) filter with rectangular weighting. An example of a selected region of interest (ROI) of raw and MA-filtered waves with marked systolic/diastolic heart peaks is in Fig. 2.

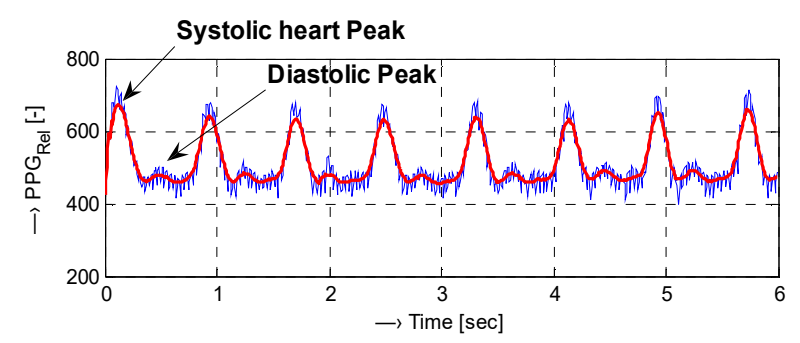

Fig. 2. Visualization of a 6-s ROI of the relative PPG signal without and with application of the 17-point MA filter, $f_{\mathrm{s}}=125 \mathrm{~Hz}$.

In practice, there exist many approaches to $\mathrm{HR}$ determination from the PPG signal. For the required realtime processing of the PPG signal, the used algorithm should be simple, rapid, and universal. Our proposed method works in four steps: (1) determination of the signal level threshold $L_{\text {THRESH, }}$ (2) two-state coding of the input signal, (3) determination of the pulse period $T_{\mathrm{HP}}$, (4) calculation of the HR values. The threshold level can be fixed for all processed PPG signals or it can be adaptive - in the frame of the calibration phase. It is activated before the start of the main PPG signal measurement. The fixed $L_{\mathrm{BASIC}}$ value is chosen so that the threshold level crosses the lower one third of the expected PPG signal range. For the adaptive setting of the threshold value $L_{\mathrm{ADAPT}}$, the peaks corresponding to the heart systolic pulses are localized first. Maximum ( $\left.L p_{\mathrm{MAX}}\right)$ and minimum $\left(L p_{\mathrm{MIN}}\right)$ levels of these peaks are used to obtain the heart pulse ripple $H P_{\mathrm{RIPP}}=\left(L p_{\mathrm{MAX}}-L p_{\mathrm{MIN}}\right) / L p_{\mathrm{MAX}} \cdot 100[\%]$. Then, the relative signal range is calculated from the offset level $\left(L_{\mathrm{OFFS}}\right)$, the mean peak level $(\mu L p)$, and the $\mathrm{A} / \mathrm{D}$ numerical range $\left(\mathrm{AD}_{\mathrm{NR}}\right)$ according to the equation $P P G_{\mathrm{RANGE}}=\left(\mu L p-L_{\mathrm{OFFS}}\right) / \mathrm{AD}_{\mathrm{NR}} \cdot 100[\%] . \quad$ Finally, the $L_{\mathrm{ADAPT}}$ value is set smaller than the smallest peak to include all heart pulses in the HR determination.

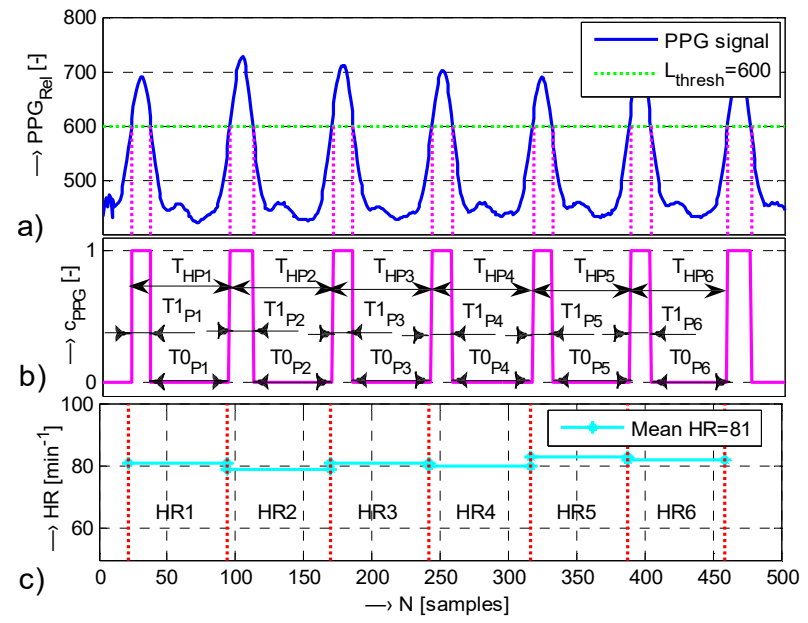

Fig. 3. An example of HR determination: a) 500-sample ROI of the PPG wave, b) clipped sequence with signed heart pulse periods $T_{\mathrm{HP}}$ from $\left.T_{1 \mathrm{P}}, T_{0 \mathrm{P}}, \mathrm{c}\right)$ partial $\mathrm{HR}$ values together with the final mean $\mathrm{HR}$.
The binary coding operation produces a sequence $c_{P P G}(i)$ with the values " 1 " or " 0 " corresponding to the input signal samples higher or lower than the chosen $L_{\text {THRESH. The heart }}$ pulse periods $T_{\mathrm{HP}}$ in samples are determined from this sequence as the length of two adjacent segments of ones $\left(T_{1 \mathrm{P}}\right)$ and zeros $\left(T_{0 \mathrm{P}}\right)$ as $T_{\mathrm{HP}}=T_{1 \mathrm{P}}+T_{0 \mathrm{P}}-$ see Fig. 3 . For the sampling frequency $f_{\mathrm{s}}$, the HR is calculated using the formula $\mathrm{HR}=60 \cdot f_{\mathrm{s}} / T_{\mathrm{HP}}\left[\mathrm{min}^{-1}\right]$. These values are next analyzed to obtain their statistical parameters

\section{PPG SENSOR REALIZATION}

Our current realization of a PPG sensor represents a low cost solution, but fully operational for our experimental purpose. On the hardware side, the control of the sensor is carried out by the Arduino Nano v. 3.0 board $^{1}$ based on the 8-bit low power processor ATmega328P running at the clock frequency $16 \mathrm{MHz}$ with eight integrated 10-bit $\mathrm{A} / \mathrm{D}$ converters. This board contains also the USB interface for direct programming and the USART module for serial communication. The second HW part is the BT module HC06 (Waveshare $4328^{2}$ ). This module enables wireless communication in the Bluetooth 2.0 standard at $2.4 \mathrm{GHz}$ with the maximum baud rate of $115200 \mathrm{bps}$. The third hardware component is represented by an optical heart rate PPG sensor (Pulse Sensor Amped - Adafruit $1093{ }^{3}$ ) working in a transmission mode with one LED source and one PHD element (see the left lower corner of the photo in Fig. 4a). The sensor printed circuit board is disk-shaped with a diameter of $16 \mathrm{~mm}$ and it includes a basic analog interface with an operational pre-amplifier. Output values of the 10-bit $\mathrm{A} / \mathrm{D}$ converter are in relative unipolar representation in the range from 0 to $1024\left(=2^{10}=\mathrm{AD}_{\mathrm{NR}}\right)$ corresponding to the input PPG signal range from 0 to $5 \mathrm{~V}$ or $3.3 \mathrm{~V}$ depending on the used type of the power supply. We tested the $5 \mathrm{~V}$ power bank supply via the USB connection, and the $3.6 \mathrm{~V}$ polymerlithium-ion (LiPo) rechargeable cell. This type of battery was chosen because it is free of any metallic components. It is very important for practical measurements - we must eliminate any interaction with the magnetic field and prevent possible disturbance in electromagnetic compatibility inside the running MRI equipment. Therefore, the processor board with the BT module and the optical sensor are covered in two separate aluminum shielding boxes as documented in Fig. 4.

The proposed PPG sensor works in the slave mode, i.e. after initialization it waits for commands from the master external device (laptop, desktop PC, etc.) via the wireless communication connection. The service program in the ATmega328P enables adjustment of the time delay $T_{\mathrm{INT}}$ for reading the analog signal from the optical sensor and the $\mathrm{A} / \mathrm{D}$ conversion. The real-time function enables only the settings: $T_{\mathrm{INT}}=\{10,8,5,4$, and $2 \mathrm{~ms}\}$ representing the sampling frequencies $f_{\mathrm{s}}=\{100,125,200,250$, and $500 \mathrm{~Hz}\}$. There are two possibilities of measurement and transmission of the PPG values: in a batch of $N_{\text {MEAS }}=\{1 \mathrm{k}, 10 \mathrm{k}$, and $25 \mathrm{k}\}$ samples, or continual measurement activated from the control application PPGsensBT. It is implemented in the Windows platform - see the screen copy in Fig. 5 This application executes the PPG signal sensing, processing, and recording in three operation modes: (1) real-time signal monitoring with simultaneous plotting of the relative PPG signal, (2) calibration mode - determination of the maximum and minimum heart peaks to estimate the adaptive threshold

\footnotetext{
1 http://arduino.cc/en/Main/ArduinoBoardNano

2 www.waveshare.com/wiki/Bluetooth Slave UART Board

3 https://pulsesensor.com/pages/code-and-guide
} 
$L_{\text {THRESH }}$ for HR calculation, (3) continual measurement of the PPG signal using the chosen $f_{\mathrm{s}}$. This measurement mode enables automatic transmission of a fixed number $N_{\text {MEAS }}$ of samples from the PPG sensor with the following two possibilities: (a) direct storing of the received PPG signal samples to a file on the disc of the master device without any further processing using the commands Start and Stop manually sent by a user, (b) storing the PPG signal samples to an internal memory buffer together with post-processing, as filtering and HR determination. The sensed PPG signals are stored in a Wave format (16-bit, mono, PCM coding).

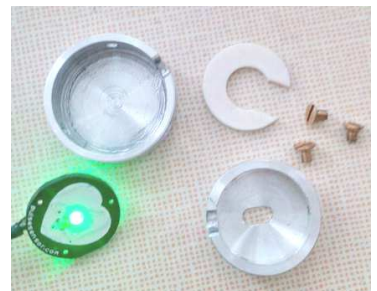

a)

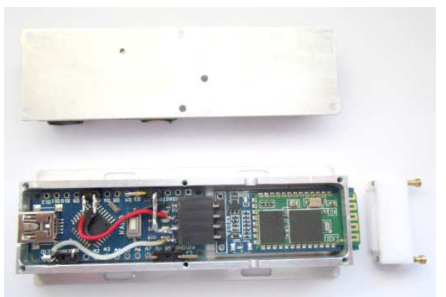

b)
Fig. 4. Assembling and aluminum covering of: a) the optical Pulse sensor, b) the body of the PPG sensor.

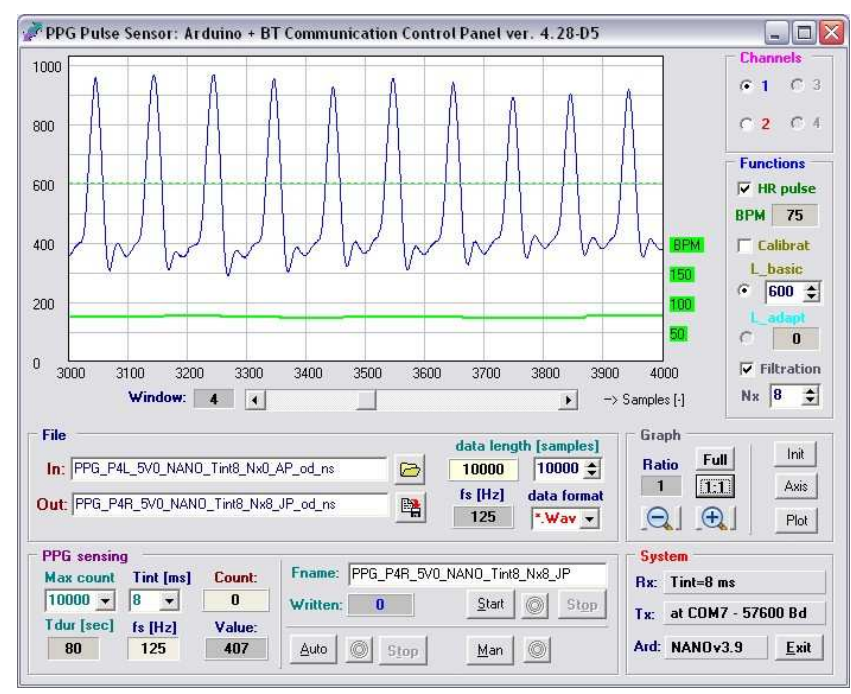

Fig. 5. Screen copy of the control application PPGsensBT.

\section{PERFORMED MEASUREMENT EXPERIMENTS}

The developed PPG sensor was tested in three phases: (1) preliminary mapping of conditions for wireless connection through the shielding cage of the MRI device, (2) basic testing and verification of functionality of sensor's structural blocks including BT communication and PPG signal sensing performed by the control application PPGsensBT in normal laboratory conditions, (3) main measurement of the PPG signals in the low magnetic field environment of the open-air MRI device E-scan Esaote Opera [7] located at our institute - see the arrangement in Fig. 6.

The preliminary testing shows, that the BT data transfer is possible because the metal cage of the tested MRI device consists of $2.5-\mathrm{mm}$ diameter holes practically enabling a wireless communication. At the same time, it enables reliable suppression of high working frequencies up to $10 \mathrm{MHz}$ from the MRI device with the basic $B_{0}=0.178 \mathrm{~T}$ [7]. The communication with the mobile device located inside the cage behind its closed door was tested with the MRI in a "silent state" (no MR sequence running): the received signal magnitude was lower but the mobile audio and data connection was still functional.

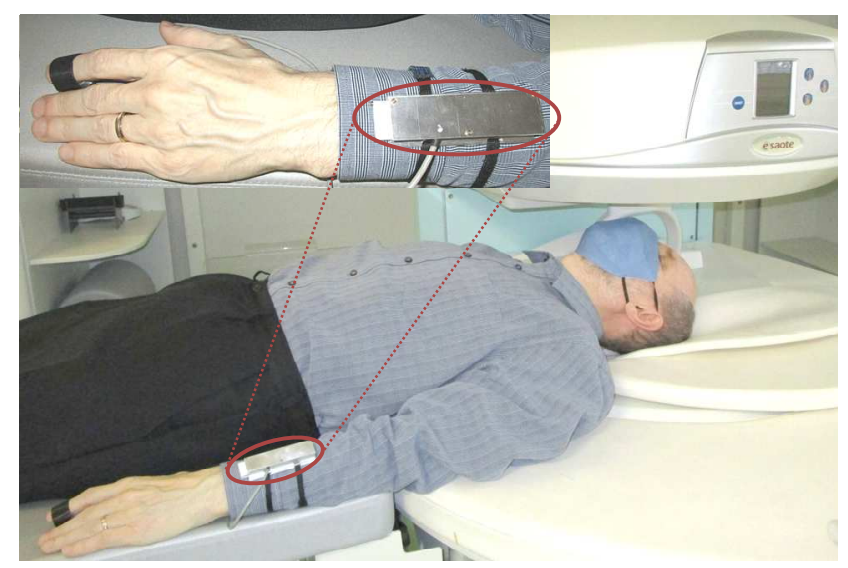

Fig. 6. Arrangement of the PPG signal measurement inside the scanning area of the open-air MRI device E-Scan Opera.

The functionality of the PPG sensor and the control application were stepwise tested in all operation modes, i.e. the real-time PPG pick-up and recording were verified using all possible sampling frequencies. The reflection optical pulse sensor was always fixed on a finger (usually forefinger) by an elastic ribbon. The tested person always sat at the table without any physical or mental activity. The baud rate of BT communication was set to maximum. The recorded PPG signals with the length of $N_{\text {MEAS }}=10 \mathrm{k}$ samples were subsequently analyzed to find dependence of the number of detected heart periods $N_{\mathrm{HP}}$ and lengths $T_{1 \mathrm{P}}, T_{0 \mathrm{P}}$ on the used sampling frequency $f_{\mathrm{s}}-$ see numerical comparison in Table I.

The main experiment consists of the PPG signal measurement with two tested volunteer healthy persons (male M1 and female F1) lying in the MRI scanning area with the MR scan sequence running or with no scanner activity. The PPG signal was picked-up with three ambient settings: (1) shielding cage door open $(O D)$ with no scan sequence $(N S),(2)$ cage door closed $(C D)$ with $N S$, (3) $C D$ with high-resolution scan sequence ${ }^{4}(S 1)$. According to the results of the auxiliary PPG signal measurements, in the main experiments the optical pulse sensor was placed on the index finger of both hands of both tested persons. The control laptop with the activated application was laid near the MRI operating console; the PPG signal pick-up and the MR scan sequence were started manually by the operator. The PPG signals were recorded immediately one after another for three tested conditions. The graphical summary comparisons of the $\mathrm{PPG}_{\mathrm{RANGE}}$ and $\mathrm{HP}_{\mathrm{RIPP}}$ parameters together with the histograms of normalized HR values (z-scores) are presented in Fig. 7.

TABLE I. INFLUENCE OF USED $F_{\mathrm{S}}$ ON DETECTED NUMBER OF HEART

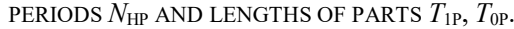

\begin{tabular}{|c|c|c|c|}
\hline $\boldsymbol{f}_{\mathrm{s}}[\mathrm{Hz}]$ & $\begin{array}{c}\boldsymbol{N}_{\mathbf{H P}} \\
{[\text { samples] }}\end{array}$ & $\begin{array}{c}\boldsymbol{T}_{\mathbf{1 P}} \\
\text { [samples] }\end{array}$ & $\begin{array}{c}\boldsymbol{T}_{\mathbf{0 P}} \\
\text { [samples] }\end{array}$ \\
\hline 100 & 87 & 11 & 76 \\
\hline 125 & 105 & 20 & 85 \\
\hline 200 & 170 & 34 & 136 \\
\hline 250 & 215 & 42 & 173 \\
\hline 500 & 409 & 83 & 326 \\
\hline
\end{tabular}

${ }^{4}$ High-resolution SE-HF sequence with setting of: $\mathrm{TE}=26 \mathrm{~ms}, \mathrm{TR}=500 \mathrm{~ms}$, sagittal orientation, slice thickness $=4.5 \mathrm{~mm}$. 


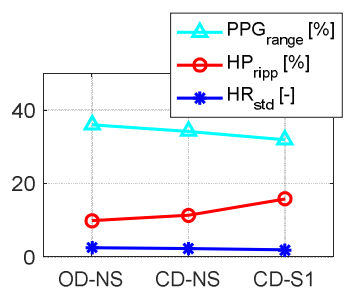

a)

Fig. 7. Summary comparison for three measurement conditions in the MRI device: a) mean values of $\mathrm{PPG}_{\mathrm{RANGE}}, \mathrm{HP}_{\mathrm{RIPP}}$, and $\mathrm{HR}_{\mathrm{STD}}$ parameters, b) histograms of z-scores calculated from HR values; both hands of both tested persons.

\section{DISSCUSION AND CONCLUSIONS}

The developed reflection PPG sensor works in real time in normal laboratory conditions for the sampling frequencies from 100 to $500 \mathrm{~Hz}$. The performed experiments confirm practical usability of the proposed sensor for continual measurement of the PPG signal in the magnetic field environment with additional $\mathrm{RF}$ and electromagnetic disturbance. The main measurements inside the MRI device show a few problems to be solved to obtain sufficient amplitude of the PPG signal, and correct HR and other parameters. To guarantee secure serial BT communication between the sensor and the control device for the decreased BT signal intensity during the shielding cage door closed the baud rate was lowered to 57600 .

From the analysis of different used sampling frequencies follows that sensing of the PPG signal with higher $f_{\mathrm{s}}(250$, $500 \mathrm{~Hz}$ ) is reasonable only for more accurate systolic pulse width determination. On the other hand, decrease of the number of detected HR periods can have a negative influence on the precision of statistical results. The setting of $f_{\mathrm{s}}=125 \mathrm{~Hz}$ was finally chosen for use in the main measurement experiment.

The measurements with the scan MR sequence executed confirmed the importance of shielding particularly parts of the PPG sensor including also the optical components with an analog interface. Here, high intensity of the MRI gradient system electromagnetic pulses affects the sensed PPG signal and the HR values are not correctly determined. Fig. 8 shows the recorded PPG wave with and without the aluminum plate shielding (see Fig. 4a). The analysis of the PPG signals recorded using the fully shielded sensor and with the applied MA filter shows that the HR accuracy is acceptable also in worse recording signal conditions. However, it is also important to analyze the influence of the BT transmission in the scanning area on the quality of the scanned MR images. To enable long-time PPG signal recording by this sensor, the acquisition should be battery saving. The wireless communication module working in BT 4.0 BLE (low energy) regime can help to solve this requirement.
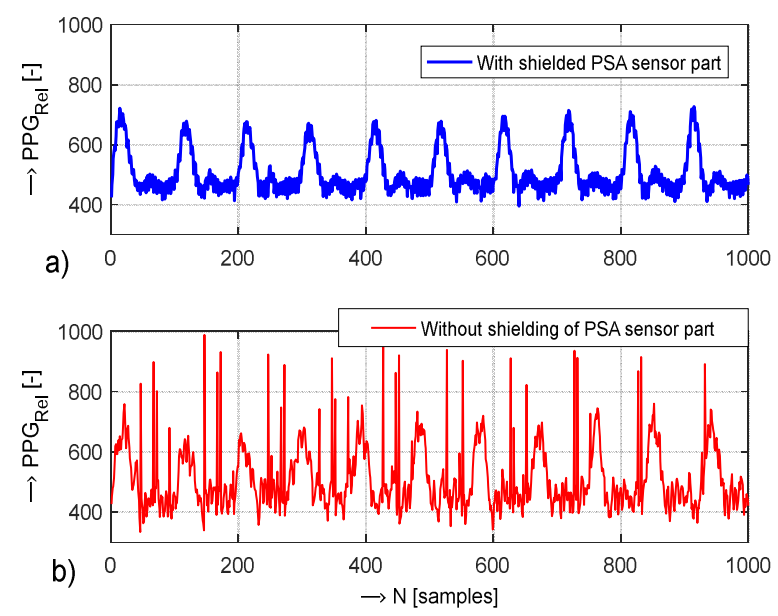

Fig. 8. Example of $1 \mathrm{k}$ sample ROI part of PPG waves sensed in the scanning area of the MRI device while the Hi-Res sequence is running: a) with and b) without shielding of the optical part of the PPG sensor; $f_{\mathrm{s}}=125 \mathrm{~Hz}$, without the MA filter.

\section{REFERENCES}

[1] N. G. Y. Aslier, Ö. Saatçi, and S. Yildiz "The effects of 1.5 Tesla contrast - Enhanced temporal bone MRI on the auditory functions and complaints of geriatric patients with tinnitus," Turk. J. Geriatr., vol. 23, pp. 230-240, May 2020.

[2] L. Schickhofer, J. Malinen, and M. Mihaescu, "Compressible flow simulations of voiced speech using rigid vocal tract geometries acquired by MRI," J. Acoust. Soc. Am., vol. 145, pp. 2049-2061, April 2019.

[3] P. Celka, P. H. Charlton, B. Farukh, P. Chowienczyk, and J. Alastruey, "Influence of mental stress on the pulse wave features of photoplethysmograms," Healthcare Technology Letters, vol. 7, pp. 712, 2020.

[4] J. Přibil, A. Přibilová, and I. Frollo, "Physiological impact of vibration and noise in an open-air magnetic resonance imager: Analysis of a PPG signal of an examined person," Proceedings, vol. 42, 2020.

[5] S. Majumder, T. Mondal, and M. J. Deen, "Wearable sensors for remote health monitoring," Sensors, vol. 17, 130, January 2017.

[6] D. Jarchi, D. Salvi, L. Tarassenko, and D. A. Clifton, "Validation of instantaneous respiratory rate using reflectance PPG from different body positions," Sensors, vol. 18, 3705, October 2018.

[7] Esaote S.p.A., E-Scan Opera. User's manual. Revision A, Genoa, 2008. 